\title{
Análise temporal de uma rede de contato hospitalar utilizando técnicas de visualização de informação
}

\author{
Cláudio D. G. Linhares ${ }^{1}$, Jean R. Ponciano ${ }^{1}$, Luis. E. C. Rocha ${ }^{2}$, \\ José Gustavo de S. Paiva ${ }^{1}$, Bruno A. N. Travençolo ${ }^{1}$ \\ ${ }^{1}$ Faculdade de Computação - Universidade Federal de Uberlândia (UFU) \\ Uberlândia - MG - Brasil \\ ${ }^{2}$ Departamento de Ciências da Saúde Pública - Instituto Karolinska, Suécia \\ \{claudiodgl, jeanrobertop\}@gmail.com, luis.rocha@ki.se \\ gustavo@ufu.br, travencolo@gmail.com
}

\begin{abstract}
The visualization of temporal networks, i.e., the visualization of networks that represent interactions between a domain's instances and that have information about when such interactions occur, plays a key role in the recognition of properties that would be difficult to perceive without an adequate visualization strategy. This paper presents an application case study of a visual analysis system in a hospital contact network between people. The goal is to demonstrate the applicability of this system in helping on decision making processes related to health data. The achieved results facilitate both the network analysis and the patterns perception, accelerating and making the decision making processes more reliable.
\end{abstract}

Resumo. A visualização de redes temporais, isto é, a visualização de redes que representam interações entre as instâncias de um domínio e que possuem informações sobre quando essas interações ocorreram, exerce um papel relevante na identificação de propriedades que seriam de difícil percepção sem uma adequada estratégia de visualização. Este trabalho apresenta um estudo de caso de aplicação de um sistema de análise visual em uma rede de contato hospitalar. $O$ objetivo é demonstrar a aplicabilidade dessa ferramenta no auxílio a tomadas de decisão em relação a dados da área da saúde. Os resultados alcançados demonstram a facilidade da análise da rede e a percepção de padrões, agilizando e deixando as tomadas de decisão mais confiáveis.

\section{Introdução}

Nos últimos anos, vários pesquisadores têm aplicado seus esforços na representação e análise de relacionamentos entre elementos em diferentes campos de estudo, como medicina, biologia, sociologia, computação e outros. A estrutura mais adotada para esse tipo de aplicação é uma rede definida por um conjunto de nós (representando as instâncias do domínio) com arestas conectando-os de acordo com algum critério de análise (por exemplo, comunicação, similaridade ou interação social) [Estrada 2015]. Nesse contexto, uma célula pode ser vista como uma rede composta por seus componentes (nós) ligados por reações químicas (arestas) [Albert and Barabási 2002]. 
Em diversas situações, no entanto, apenas conhecer as instâncias e saber das ocorrências de conexões entre elas pode não ser suficiente para representar e analisar as relações existentes na rede. Nesses casos, conhecer também os instantes de tempo em que tais interações ocorreram torna-se essencial para uma análise mais precisa. Por exemplo, no campo de estudo da epidemiologia pode-se usar uma estrutura de rede em que cada nó representa uma pessoa, as arestas conectam as pessoas (i.e., os nós) que possuem algum tipo de relação (por exemplo, de proximidade física) e os instantes de tempo indicam quando as interações entre as pessoas ocorreram (por exemplo, um aperto de mão ou uma conversa próxima). Uma rede que dispõe dessas três informações recebe diferentes nomes na literatura, como redes dinâmicas, redes temporais (nome adotado neste trabalho) e outros [Holme and Saramäki 2012].

A análise manual das redes temporais pode dificultar a detecção de alguns padrões, tendências, anomalias e outros fenômenos devido à quantidade de informações. O estudo da visualização da informação busca facilitar a análise por meio de abordagens computacionais que sejam gráficas e interativas. Assim, estratégias adequadas de visualização de redes temporais são importantes para o correto entendimento das interações entre os nós e da evolução da rede ao longo do tempo. Apesar de ser uma área de estudo em expansão, a exploração visual de redes temporais ainda é um problema não-resolvido e que necessita de abordagens efetivas [Linhares et al. 2017].

Este artigo apresenta um estudo de caso de análise visual de uma rede de contatos hospitalar utilizando o sistema computacional DyNetVis [Linhares et al. 2017]. Esse sistema apresenta diversos layouts que organizam as informações da rede em estruturas visuais, de forma a ressaltar padrões que representem fenômenos associados. Além disso, oferece diversas ferramentas interativas que auxiliam na exploração de padrões visuais em redes temporais. Por meio desse estudo de caso, pretende-se demonstrar a aplicabilidade do sistema e de seus métodos na análise de redes com dados da área da saúde, incluindo dados para gestão de unidades hospitalares e equipes médicas, dentre outros.

O restante deste artigo está dividido como se segue. A Seção 2 apresenta estratégias de visualização de redes temporais. A Seção 3 apresenta a ferramenta computacional DyNetVis. A Seção 4 descreve em detalhes o estudo de caso realizado. Por fim, a Seção 5 traz as conclusões e perspectivas de trabalhos futuros.

\section{Visualização de Redes Temporais}

A visualização de redes temporais permite a análise de padrões, tendências, anomalias e outros tipos de comportamentos existentes em dados reais que possuem informação temporal. A Figura 1 ilustra três formas de visualização para uma mesma rede temporal fictícia. A primeira forma, apresentada na Figura 1(a), representa os dados em forma tabular. Nesse tipo de visualização, a percepção de padrões é dificultada, em especial quando ela possui um grande volume de informações, visto que toda a rede é representada textualmente. Esse mesmo conjunto tabular de dados pode ser representado em uma estrutura clássica de grafos, conforme mostrado na Figura 1(b). Neste trabalho, esse tipo de visualização é chamado de visão estrutural da rede. Apesar de facilitar a análise global dos dados, essa abordagem dificulta a análise temporal da rede, pois não exibe os momentos em que as interações entre as instâncias ocorreram. Nesse contexto, surge a estratégia chamada de visão temporal (Figura 1(c)), que destaca os instantes de tempo, facilitando 
a percepção da evolução da rede. Essa característica torna a visualização temporal útil para análises epidemiológicas, além de ser também adequada em outros contextos. Tanto a visão estrutural quanto a temporal, apresentadas na Figura 1, são descritas em detalhes nas próximas seções.

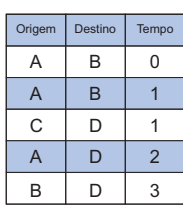

(a)

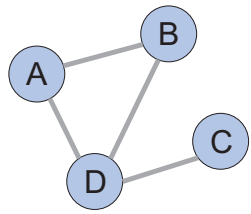

(b)

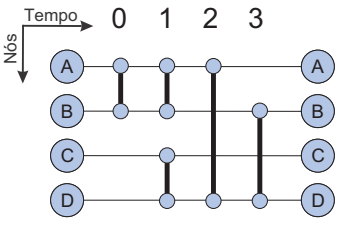

(c)

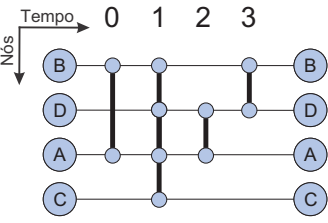

(d)

Figura 1. Tipos de visões dos dados: (a) representa os dados em forma de tabela; (b) representa a visão estrutural dos dados; em (c) os dados são exibidos na visão temporal. Nesse tipo de visão, a ordem em que os nós são dispostos no eixo vertical impacta diretamente no resultado da visualização da rede; (d) apresenta um novo layout temporal gerado pela reordenação da sequência de nós exibida em (c).

\subsection{Visão Estrutural}

A visão estrutural é descrita como a forma convencional de um grafo com instâncias (representadas por nós) dispostas espacialmente com conexões (arestas) ligando-as. Desse modo, um grupo de pessoas interagindo pode ser representado por uma visão estrutural se considerarmos que cada nó representa uma pessoa e que as arestas ligam duas pessoas que interagiram entre si. Esse tipo de visão é mais adequada para analisar a rede como um todo, facilitando a percepção de padrões globais que independem dos instantes de tempo em que as conexões ocorreram. É possível visualizar, por exemplo, quais nós apresentam grande número de conexões e quais pares de nós possuem mais conexões entre si.

\subsection{Visão Temporal}

A visão temporal baseia-se em um layout que utiliza o eixo das abscissas para representar o tempo e o eixo das ordenadas para representar os nós da rede. Nessa visão, as arestas indicando as conexões são retas verticais ligando dois nós em determinados instantes de tempo, como visto na Figura 1(c). Devido a essa característica, ela permite analisar a rede de acordo com sua evolução ao longo do tempo [Holme and Saramäki 2012]. Esse tipo de visão é a base para o entendimento deste trabalho.

A posição dos nós na visão temporal tem muita influência na visualização gerada e, consequentemente, na percepção dos padrões da rede. As Figuras 1(c) e 1(d) ilustram dois exemplos em que um mesmo conjunto de dados gera duas visões temporais diferentes devido à alteração da ordem dos nós. Isso ocorre pois quanto mais distantes dois nós estão, maior a aresta que representará a conexão entre eles. Arestas grandes são prejudiciais na medida em que causam sobreposição em outras, possivelmente ocultando comportamentos relevantes na análise dos dados. Nesse sentido, é importante organizar os nós em uma sequência que deixe o layout menos denso, facilitando a análise. Em [van den Elzen et al. 2013] são apresentados vários métodos de reordenação de nós. Três métodos da literatura são descritos a seguir [van den Elzen et al. 2013, Linhares et al. 2017]: 
1. Nascimento: Nesta ordenação, os nós são inseridos na ordem em que se conectam a algum outro nó pela primeira vez, ou seja, quando "nascem". Dessa forma, os nós que aparecem nos primeiros instantes de tempo serão posicionados na parte superior do layout e os últimos nós que surgirem serão posicionados na porção inferior.

2. Lexicográfico: Nesta ordenação, os nós são inseridos em ordem crescente de rótulo, o qual varia de acordo com o domínio do problema. Se os nós são rotulados com números, a ordenação é numérica crescente; se forem letras ou palavras, a ordem será alfabética.

3. Vizinhos Recorrentes: Esta ordenação, proposta em [Linhares et al. 2017], centraliza e aproxima os nós que mais se conectam entre si, ou seja, os nós que são vizinhos (adjacentes) na rede. No primeiro passo do algoritmo, o nó com mais conexões é inserido no centro do layout. Ao redor dele são colocados então os nós que mais se conectam a ele. O processo continua recursivamente tentando aproximar espacialmente os nós que mais se conectam entre si.

Outra estratégia de visualização temporal, ideal para identificar o nível de intensidade das atividades dos nós em instantes de tempo específicos, além de outros padrões globais, é conhecida como Mapa de Atividade Temporal (TAM - Temporal Activity Map) [Linhares et al. 2017]. Trata-se de uma técnica que oculta as arestas e dá destaque para a exibição dos nós, associando, para cada nó e em cada instante de tempo, uma cor baseada em uma escala de cores que visa refletir o nível de interações do nó naquele instante. Com essa estratégia, consegue-se detectar, por exemplo, em quais momentos há gargalos e horários de pico, informações relevantes para suportar decisões gerenciais.

\section{DyNetVis}

O Sistema DyNetVis (Dynamic Network Visualization) [Linhares et al. 2017] exibe as informações da rede utilizando as estratégias visuais apresentadas anteriormente. Ele também oferece diversas ferramentas para auxiliar a análise visual e o reconhecimento de padrões visuais, como seleção coordenada entre visões, zoom-in e zoom-out, personalização de cores e tamanhos de nós e arestas e ainda apresenta diversas opções de disposição de nós tanto estruturalmente quanto temporalmente.

O sistema subdivide suas funcionalidades em duas telas principais, contendo as visões estrutural (Figura 2(a)) e temporal (Figura 2(b)). Dentre as funcionalidades oferecidas na visão estrutural, destacam-se a seleção de nós, adequada para análises de instâncias específicas da rede, e a possibilidade de modificação de propriedades dos nós e arestas. A análise da visão estrutural pode ser prejudicada se a rede possuir muitos nós devido à potencial sobreposição deles. Por esta razão, o DyNetVis permite livre reorganização espacial dos nós pelo usuário, manualmente ou por meio de técnicas como algoritmos de força [Zhang et al. 2005], circular [Baur and Brandes 2005] e hierárquico [Bastert and Matuszewski 2001].

$\mathrm{Na}$ visão temporal tem-se novamente a possibilidade de modificação de propriedades dos nós e arestas, bem como as opções de alterar a ordenação dos nós, permitindo que o usuário escolha e altere sempre que julgar conveniente, uma das ordenações disponíveis (apresentadas na Seção 2.2). Há ainda, nesse layout, a possibilidade de aplicar a visualização TAM, ideal para análise do nível de atividade dos indivíduos ao longo do 
tempo. Algumas funcionalidades são coordenadas em ambas as visões, como a seleção de nós, tendo assim uma percepção do comportamento dos mesmos nós em duas visões diferentes. Essa situação é apresentada na Figura 2, em que ambas as telas do sistema destacam um conjunto de nós selecionado.
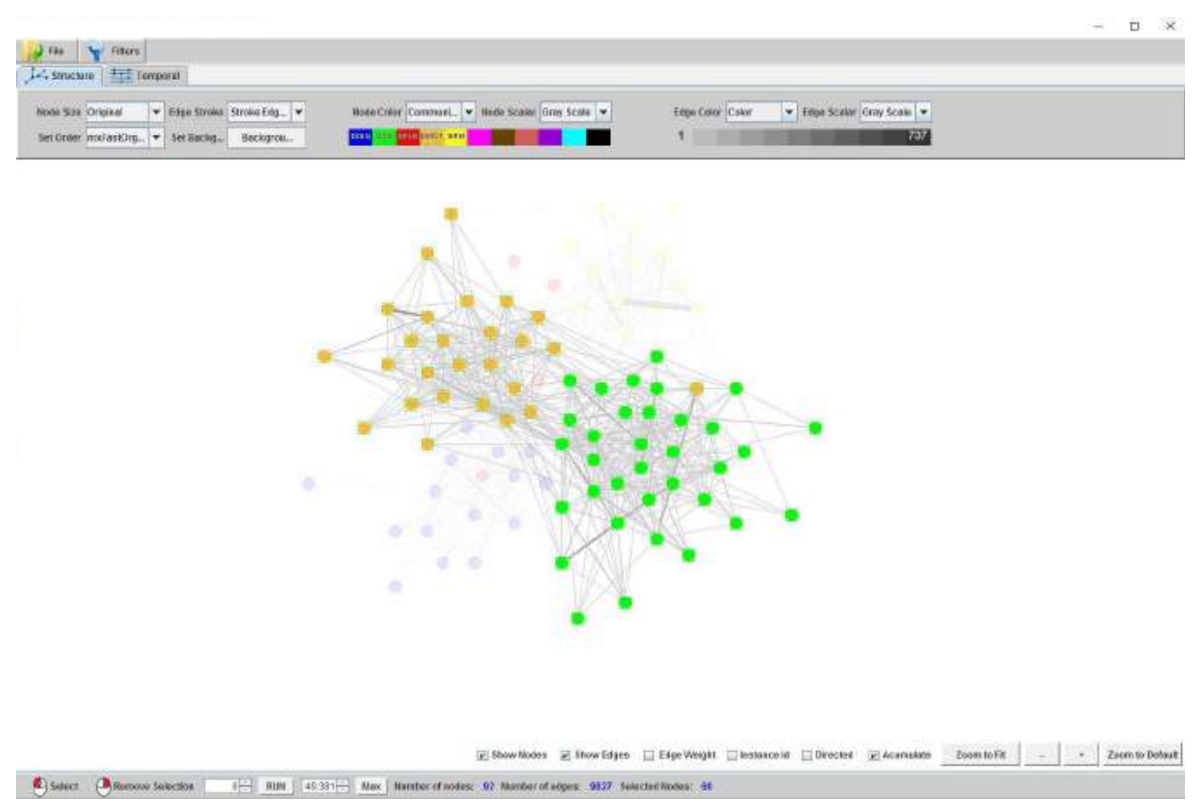

(a) Visão Estrutural

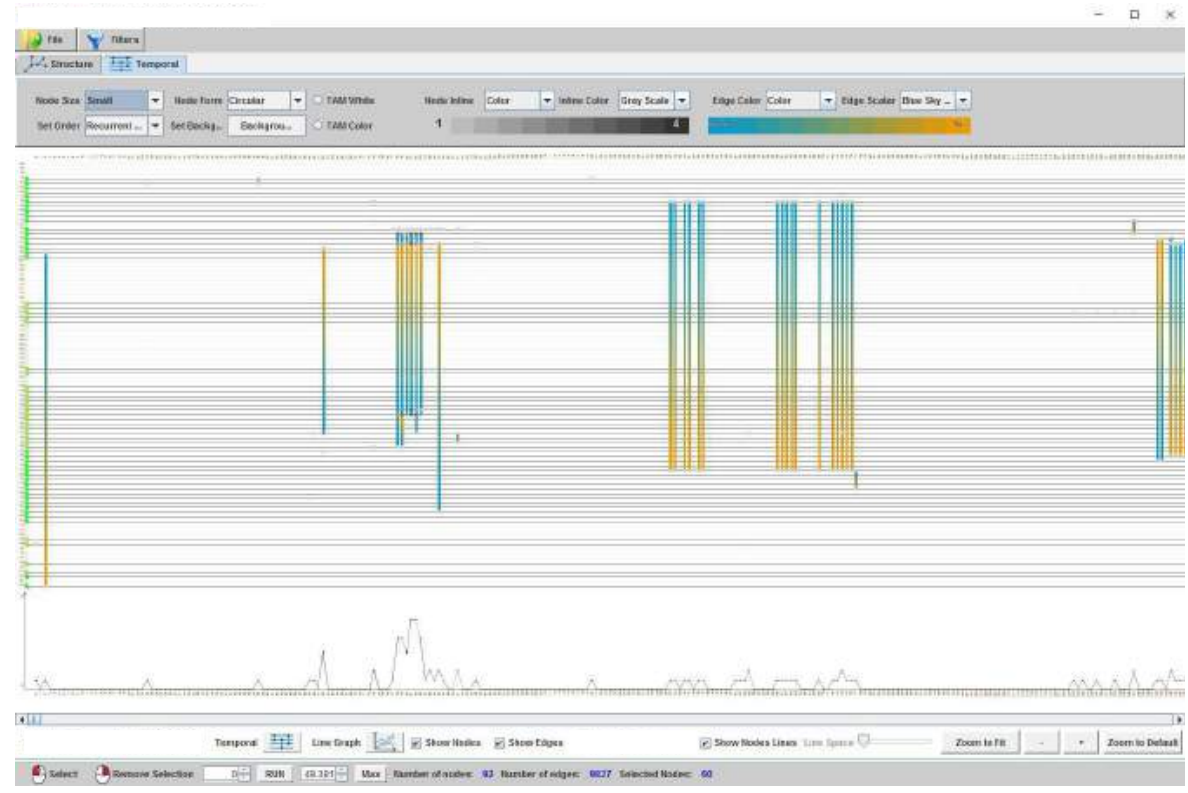

(b) Visão Temporal

Figura 2. As duas principais telas do sistema DyNetVis para exploração estrutural e temporal das informações da rede. (a) Visão estrutural. (b) Visão Temporal. Os nós destacados em (a) também são destacados em (b), ilustrando a coordenação na seleção de nós. 


\section{Estudo de Caso}

Esta seção apresenta um estudo de caso elaborado utilizando uma rede de contatos entre pessoas (também conhecida como rede de proximidade) com informações provenientes de um hospital. O objetivo deste estudo é demonstrar a aplicabilidade do sistema DyNetVis, aliado ao método de reordenação de nós Vizinhos Recorrentes, na análise de tendências, periodicidades, anomalias e outros padrões existentes em redes temporais.

\subsection{Rede de contato - Hospital}

Todas as informações constantes na rede foram coletadas por meio de sensores RFID. Quando ocorre o contato por aproximação frontal entre duas pessoas distanciadas entre si de 1 até 1,5 metros, o contato é registrado. Dois indivíduos fazem contato se houver pelo menos uma troca de informações entre seus dispositivos RFID em um intervalo de 20 segundos. De igual forma, considera-se que o contato é interrompido quando não há troca de informações nesse intervalo [Cattuto et al. 2010, Vanhems et al. 2013].

Os dados da rede Hospital [Vanhems et al. 2013] foram coletados em uma unidade geriátrica de um hospital universitário com quase 1000 leitos existente em Lyon França. Os dados colhidos são relativos a cinco dias consecutivos, começando às 13 horas do dia 06 de dezembro de 2010 (segunda-feira) e encerrando-se às 14 horas do dia 10 de dezembro do mesmo ano (sexta-feira). Os dados são relativos a 46 funcionários (92\% de participação) e 29 pacientes (94\% de participação). Dos 46 funcionários, 11 são médicos (perfil MED), 27 são enfermeiros ou auxiliares de enfermagem (perfil ENF) e 8 são funcionários administrativos (perfil ADM). Ao grupo de pacientes foi associado o perfil PAC. Os participantes foram monitorados durante todo o tempo em que permaneceram no hospital. Os visitantes não foram considerados na coleta dos dados. No total, a rede conta com 75 indivíduos (nós) e 32.424 contatos (conexões) distribuídos nos cinco dias.

\subsection{Análise Visual}

A fim de tornar o reconhecimento de padrões visuais tão fácil quanto possível nos experimentos a seguir, duas decisões de layout foram adotadas. Inicialmente, baseado no princípio da similaridade de Gestalt [Ware 2013], que afirma que a visão humana tende a agrupar objetos similares, foram associadas cores diferentes para os nós de perfis diferentes na rede. Essa associação é visível tanto na visão estrutural quanto na temporal e facilita a identificação dos perfis, agilizando o reconhecimento de comportamentos e tomadas de decisão. A segunda decisão de layout diz respeito às cores das arestas na visão temporal: é associada às arestas uma escala de cores que varia de azul até laranja, representando a origem e o destino da conexão, respectivamente. A importância dessa decisão surge em situações nas quais o sentido do contato é importante para análise, como, por exemplo, em situações epidemiológicas, em que é importante saber quem infectou quem com determinada doença.

A Figura 3 apresenta o layout estrutural gerado pelo DyNetVis para a rede do Hospital. Os nós estão separados por um algoritmo baseado em força, que posiciona os nós com mais conexões na região central do grafo e os nós com poucas conexões na região periférica dele. Assim, é possível ver, por exemplo, que os pacientes possuem, no geral, pouco contato tanto com membros de outros perfis quanto entre si - o 
que é justificável pelo fato de a maioria dos quartos (todos, exceto dois) serem individuais [Vanhems et al. 2013]. Por outro lado, os enfermeiros e assistentes de enfermagem estão majoritariamente dispostos no centro da rede, indicando alto grau de conectividade desses profissionais. A visão estrutural não permite visualizar quando os contatos entre os indivíduos ocorreram. Por essa razão, é importante também uma análise temporal da rede.

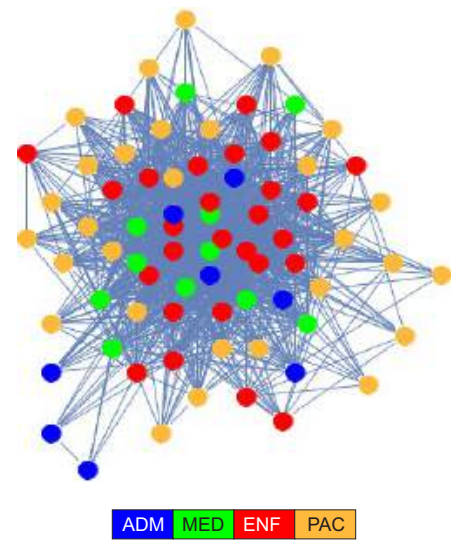

Figura 3. Visão estrutural da rede Hospital, com nós coloridos de acordo com o perfil de cada participante da rede (MED - médicos, ENF - enfermeiros ou auxiliares, ADM - funcionários administrativos, PAC - pacientes). A disposição dos nós no layout foi feita por um algoritmo baseado em força, o qual mantém os nós com mais conexões no centro do grafo. É possível ver que os nós que representam os pacientes encontram-se na região periférica da rede, o que indica pouco contato envolvendo os membros desse perfil.

$\mathrm{Na}$ visão temporal, a ocorrência de arestas muito grandes no layout tende a prejudicar a percepção de padrões, já que elas podem deixar o layout muito denso, ocultando regiões importantes devido a sobreposições de arestas e levando o usuário a uma possível tomada de decisão equivocada. Desse modo, é extremamente importante que o layout exiba nós com muitas conexões entre si próximos uns dos outros, a fim de reduzir o tamanho das arestas entre eles e tornando o layout tão limpo quanto possível.

Com o objetivo de analisar a qualidade do layout temporal proporcionado por diferentes técnicas de reordenação de nós, a Figura 4 apresenta um comparativo das estratégias de ordenação de nós (1) Nascimento, (2) Lexicográfico, e (3) Vizinhos Recorrentes. É possível notar que o layout proporcionado pelo último método é, de modo geral, mais limpo e portanto mais adequado para a análise da rede. Dessa forma, os experimentos seguintes foram realizados utilizando apenas a técnica Vizinhos Recorrentes e são válidos para demonstrar a relação existente entre nós posicionados próximos pelo método. A Figura 4 também permite visualizar o comportamento geral da rede nos períodos diurno e noturno. Com exceção da segunda noite, todas as outras apresentam atividade, ainda que pouca. Isso é esperado, já que a rede representa um hospital e, portanto, possui enfermeiros e pacientes também no período noturno.

A Figura 5 apresenta as interações envolvendo um paciente (nó 1383), um médico (nó 1157) e um enfermeiro (nó 1114) ao longo dos três primeiros dias na rede. Os nós na cor cinza indicam que aquele indivíduo está ativo naquele instante de tempo, ou seja, ele apresenta conexões na rede, porém não com os nós que estão destacados no layout. Por 


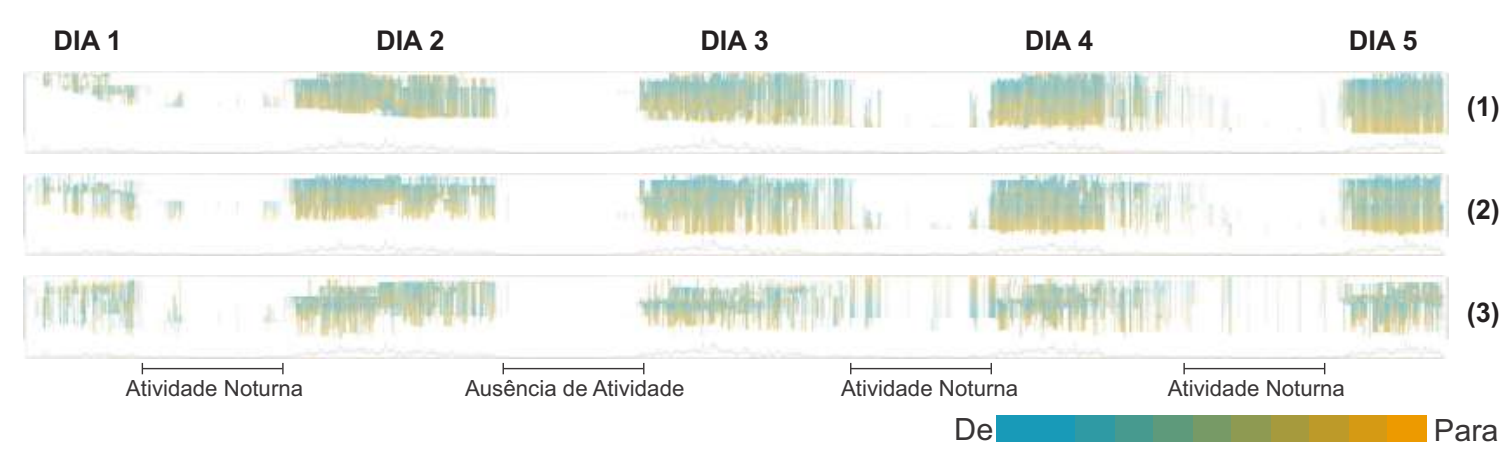

Figura 4. Comparativo entre as visões temporais obtidas por três métodos de ordenação de nós considerando os cinco dias da rede. Visualizações obtidas utilizando os métodos (1) Nascimento; (2) Lexicográfico; (3) Vizinhos Recorrentes. Cada instante de tempo nos layouts refere-se a um intervalo de três minutos e considera todas as conexões que ocorreram nesse intervalo.

essa razão, esses nós aparecem mesmo quando não existem conexões com os demais nós analisados. No dia 1, apesar de os três indivíduos estarem presentes na rede, não houve interações entre eles, situação percebida pela ausência de arestas na visualização. No segundo dia, é possível visualizar várias interações entre os indivíduos, sendo a primeira delas referente ao contato entre o enfermeiro e o paciente (aresta entre os nós 1383 e 1114). Na sequência, o enfermeiro entrou em contato com o médico e retornou ao paciente, o que permite supor que o enfermeiro entrou em contato com o médico para tratar de assuntos relacionados ao estado de saúde deste paciente. É possível observar ainda que, algum tempo depois, o enfermeiro voltou a entrar em contato com o médico e novamente com o paciente (arestas no centro da Figura 5, dia 2). Imediatamente após essas novas interações, o médico entrou em contato com o paciente pela primeira vez, repetindo o contato duas vezes em um curto espaço de tempo nesse segundo dia e outras quatro vezes ao longo do terceiro dia. Em um cenário de propagação de alguma doença, a visualização temporal, por permitir que se conheça a sequência das conexões e os indivíduos envolvidos nelas, pode ser utilizada para rastrear a infecção e prever comportamentos, possibilitando assim que sejam adotadas medidas de contenção adequadas.

DIA 1:

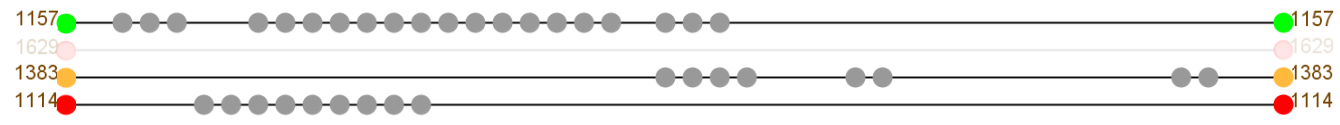

DIA 2:

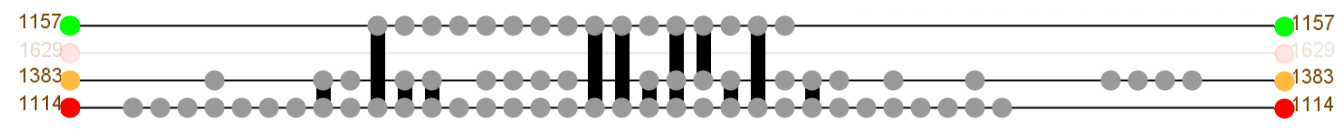

DIA 3:

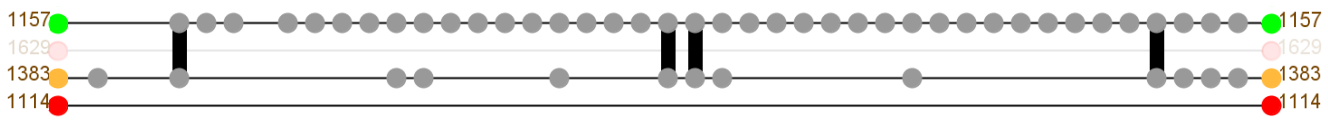

MED ENF PAC

Figura 5. Visão temporal mostrando o relacionamento entre um médico, um enfermeiro e um paciente ao longo dos três primeiros dias da rede. Cada instante de tempo na imagem refere-se a um intervalo de quinze minutos e considera todas as conexões nesse intervalo como uma só. 
A visualização temporal da rede utilizando o mapa de atividade temporal (TAM) facilita e torna mais rápida a percepção de determinados padrões globais. A Figura 6 ilustra um exemplo, aliando esse tipo de visualização com a seleção de nós, por meio do qual é possível perceber comportamentos e tomar decisões baseando-se neles. Analisando a imagem, percebe-se, por exemplo, que (1) há horário de expediente bem definido para o conjunto de médicos selecionado; (2) esse expediente é igual para todos os médicos, de modo que não há interações envolvendo esse perfil no período noturno, o que permite supor que não há plantonistas neste período; (3) em cada dia houve ausência de pelo menos um médico desse conjunto, isto é, não houve um dia sequer em que todos eles estivessem presentes. A mesma escala de cores adotada nas arestas da visão temporal foi aplicada no TAM de modo a refletir a intensidade de contatos que os profissionais tiveram ao longo dos dias (quanto mais alaranjado um bloco é, mais contatos aquele médico teve em determinado intervalo de tempo - meia hora, neste experimento). No contexto desta rede, essa informação pode ser utilizada para detectar gargalos e adequar a alocação da equipe médica em momentos de pico.

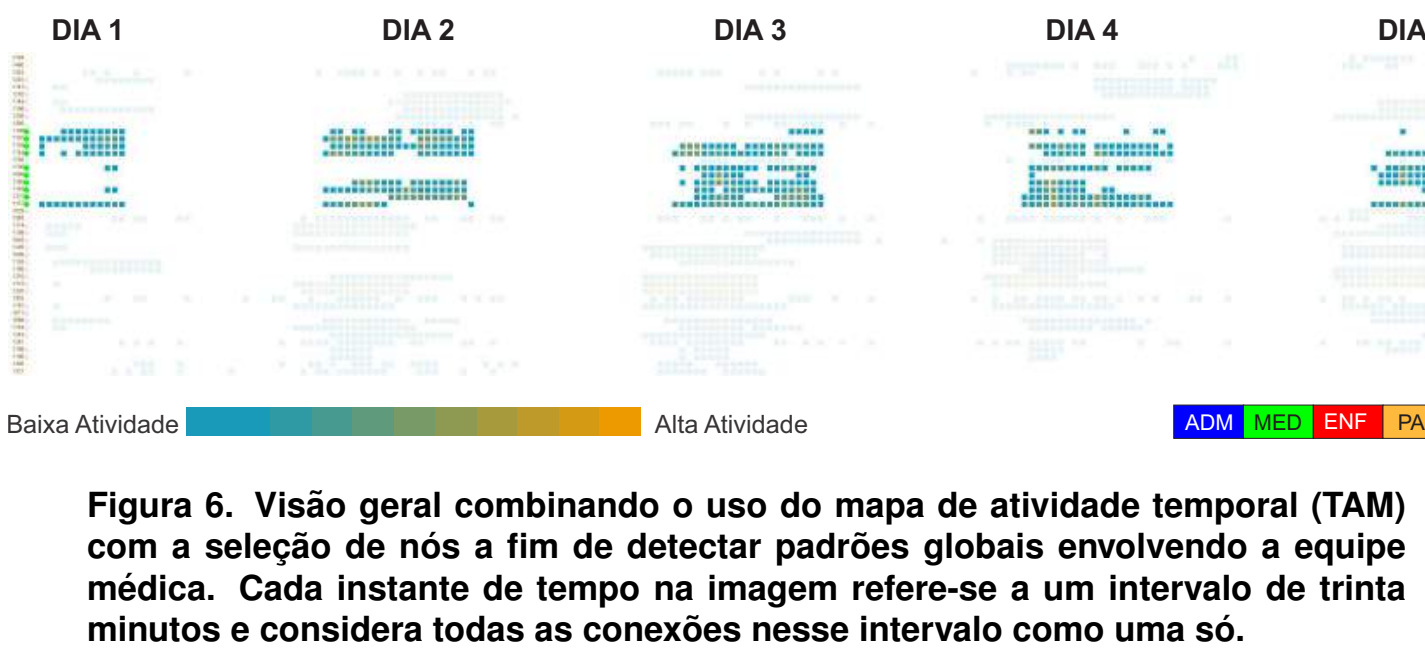

\section{Conclusão e Trabalhos Futuros}

A ferramenta DyNetVis possibilita a análise de padrões visuais em redes temporais de qualquer natureza, sendo adequada, conforme demonstrado ao longo deste artigo, para análises de redes de dados da área da saúde. Foi apresentado um estudo de caso de uma rede hospitalar utilizando esse sistema, em especial o método de ordenação de nós Vizinhos Recorrentes e a estratégia de visualização TAM, ambos presentes na ferramenta. Como resultado, foram detectados diversos padrões visuais, alguns globais, como a detecção de baixa atividade no período noturno na rede e horário de trabalho dos médicos, além de padrões mais específicos, como a relação entre paciente, médico e enfermeiro discutida na Seção 4.2. Esses resultados possibilitam tomadas de decisão mais ágeis e confiáveis. Além disso, eles demonstram a possibilidade de aplicar a ferramenta e técnicas em cenários de detecção e propagação de doenças entre indivíduos da rede.

Como trabalhos futuros é possível incorporar técnicas para aplicação em cenários de detecção epidemiológica, bem como simulações de pessoas doentes e imunes na rede e o seu impacto geral nessa propagação. Além disso, pretende-se analisar outros cenários envolvendo saúde e incluir medidas estatísticas de redes, tais como centralidade e propriedades topológicas, para realçar outros padrões. 


\section{Agradecimentos}

Este trabalho é apoiado pelo Conselho Nacional de Desenvolvimento Científico e Tecnológico - CNPq (processo 456855/2014-9); Coordenação de Aperfeiçoamento de Pessoal de Nível Superior (Capes) e Fundação de Amparo à Pesquisa do Estado de Minas Gerais - FAPEMIG (processos APQ-01345-13, REONCO-52-11).

\section{Referências}

Albert, R. and Barabási, A.-L. (2002). Statistical mechanics of complex networks. Rev. Mod. Phys., 74(1):47-97.

Bastert, O. and Matuszewski, C. (2001). Layered drawings of digraphs. In Kaufmann, M. and Wagner, D., editors, Drawing Graphs, volume 2025 of Lecture Notes in Computer Science, pages 87-120. Springer Berlin Heidelberg.

Baur, M. and Brandes, U. (2005). Crossing reduction in circular layouts. In Hromkovič, J., Nagl, M., and Westfechtel, B., editors, Graph-Theoretic Concepts in Computer Science, volume 3353 of Lecture Notes in Computer Science, pages 332-343. Springer Berlin Heidelberg.

Cattuto, C., Van den Broeck, W., Barrat, A., Colizza, V., Pinton, J.-F., and Vespignani, A. (2010). Dynamics of person-to-person interactions from distributed rfid sensor networks. PloS one, 5(7):e11596.

Estrada, E. (2015). Introduction to Complex Networks: Structure and Dynamics, pages 93-131. Springer International Publishing, Cham.

Holme, P. and Saramäki, J. (2012). Temporal networks. Physics Reports, 519(3):97 125.

Linhares, C. D. G., Travençolo, B. A. N., Paiva, J. G. S., and Rocha, L. E. C. (2017). Dynetvis: A system for visualization of dynamic networks. Symposium on Applied Computing, pages 187-194.

van den Elzen, S., Holten, D., Blaas, J., and van Wijk, J. J. (2013). Reordering massive sequence views: Enabling temporal and structural analysis of dynamic networks. In Visualization Symposium (PacificVis), 2013 IEEE Pacific, pages 33-40. IEEE.

Vanhems, P., Barrat, A., Cattuto, C., Pinton, J.-F., Khanafer, N., Régis, C., Kim, B.-a., Comte, B., and Voirin, N. (2013). Estimating potential infection transmission routes in hospital wards using wearable proximity sensors. PLoS One, 8:e73970.

Ware, C. (2013). Information Visualization: Perception for Design, volume 3. Morgan Kaufmann Publishers Inc.

Zhang, Q.-G., Liu, H.-Y., Zhang, W., and Guo, Y.-J. (2005). Drawing undirected graphs with genetic algorithms. In Wang, L., Chen, K., and Ong, Y., editors, Advances in Natural Computation, volume 3612 of Lecture Notes in Computer Science, pages 2836. Springer Berlin Heidelberg. 